\title{
A prospective study of the effect of video games on robotic surgery skills using the high-fidelity virtual reality RobotiX simulator
}

This article was published in the following Dove Press journal:

Advances in Medical Education and Practice

\section{Andreas Pierre Hvolbek' Philip Mørkeberg Nilsson ${ }^{2}$ \\ Francesco Sanguedolce ${ }^{3}$ Lars Lund ${ }^{1,3,4}$}

'Department of Urology, Odense University Hospital, Odense, Denmark; ${ }^{2}$ Copenhagen Academy for Medical Education and Simulation, Rigshospitalet, University of Copenhagen and the Capital Region of Denmark, Copenhagen, Denmark; ${ }^{3}$ Department of Urology, Aalborg University Hospital, Aalborg, Denmark; ${ }^{4}$ Department of Clinical Research, University of Southern Denmark, Odense, Denmark
Correspondence: Lars Lund Department of Urology, Odense University Hospital, J.B. Winsloew Vej 4, Entrance 20, Penthouse, 2. Floor, Odense C DK-5000, Denmark

Tel +45 5 I 408982

Email Lars.Lund@rsyd.dk
Background: Robot-assisted surgery is a growing field. Prior video game experience might give advantage to novice robotic surgeons.

Aim: Assessing if prior video gaming experience gives advantage in performing highfidelity virtual reality (VR)-simulated robotic surgery.

Methods: In this observational study, 30 medical students and 2 interns (17 females; 15 males) with median age 25 years (range, 24-26 years) were recruited and subsequently divided into groups according to prior gaming experience; gamers ( $\geq 6$ video game hours/ week) vs nongamers ( $<6$ video game hours/week). Participants performed VR-simulated urethrovesical anastomosis on RobotiX Mentor, which measured performance parameters. Participants answered a questionnaire for demographics and gaming experience. Groups were compared using Mann-Whitney $U$ and multiple regression.

Results: Gamers significantly outperformed nongamers in 3 of 24 performance metrics $(p<0.05)$, and there was a trend toward better results for 7 of the 21 remaining metrics. Males outperformed females in 5 of 24 metrics $(p<0.05)$ but were overrepresented among gamers.

Conclusion: Prior video game experience $>6 \mathrm{hrs} /$ week might give advantage in simulated robotic surgery. We recommend future studies testing this hypothesis to develop simulator programs for certification of robotic surgeons.

Keywords: robot-assisted surgery, procedure specific, virtual reality, computer simulation, video game

\section{Introduction}

Robot-assisted surgery has grown through the last decades and will most likely continue this growth in the future. The field involves a number of specialities including urology, cardiothoracic surgery, gynecology, general surgery and otorhinolaryngology and is still expanding. ${ }^{1}$ Performing robotic surgery using the da Vinci ${ }^{\circledR}$ Surgical System (Intuitive Surgical, Sunnydale, CA, USA) has proven to cause less complications (eg, reduced blood loss and shorter hospital stay) than traditional laparoscopic surgery. ${ }^{2}$ However, the steep learning curve held together with the still rising awareness of patient safety, reduced working hours and financial constraints in health care organizations causes the need for training outside the operating theater. ${ }^{3}$

Previous studies suggest that video game players have better baseline laparoscopic skills and that video games could be a way to train laparoscopic skills in surgical residents. ${ }^{4}$ Both observational studies discussing the effect of prior gaming 
experience and controlled experiments using video games as an intervention have found an advantage of playing video games on laparoscopic skills. When testing on virtual reality (VR) and box-trainer, prior and recent video game experiences have both been associated with better baseline laparoscopic skills, but when testing on porcine models, there has not been identified any advantage with prior or recent video game experience. ${ }^{4}$

The latest robotic VR simulator, RobotiX Mentor (RM) (3D Systems, Simbionix Products, Cleveland, OH, USA), offers high-fidelity procedure-specific simulations for hysterectomy, lobectomy, vaginal cuff closure, inguinal hernia repair and prostatectomy using the da Vinci interface. ${ }^{5}$ These procedure-specific 3D simulations provide a very realistic platform to practice procedural tasks safely in a VR environment. ${ }^{7}$ Since robotic naive will never be allowed to perform real-time surgery, VR simulation might offer the most authentic scenario to test robotic skills besides the more expensive porcine models and human cadavers. VR simulators have the benefit of an inherent objective performance evaluation report, whereas when testing on physical models the presence of an observer to evaluate performance is needed. ${ }^{5}$

The development of video games is a steep and everchanging process; lately, we have seen VR playing a greater role in the gaming world. VR resembles the intuitive interface seen in the da Vinci robot and so they might improve the psychometric skills necessary for robotic surgery. If so, video games might be incorporated into programs for training and certification of future robotic surgeons.

Other studies have assessed the effect of video game experience on simulated robotic surgery skills. ${ }^{6-8}$ However, doing robotic surgery requires many other skills. Therefore, it would be of interest to investigate the effect of video game experience on more complex tasks in robotic surgery, eg, suturing an anastomosis on a high-fidelity simulator.

\section{Methods}

\section{Study design and participants}

This prospective, observational, comparative and multiinstitutional study took place in Aalborg University Hospital and at Copenhagen Academy for Medical Education and Simulation located at Rigshospitalet in Copenhagen, Denmark.
We obtained written informed consent from robotic naive participants who were recruited locally in the period between January and April 2017. It was not necessary to get an approval from a review board or ethics committee. We used a sample size of convenience; 30 medical students and 2 interns were recruited and subsequently categorized into groups according to prior video game play in hours/week (eg, computer, PlayStation, Wii and X-box). Participants were divided into two groups according to prior video game experience and tested for a difference in the performance of a simulated urethrovesical anastomosis. The students or interns have never participated or assisted in laparoscopy or robotic surgery.

Exclusion criteria were experience with VR simulation in robotic surgery and prior performance of robotic surgery as these might affect the performance outcome; for the same reason, we did not include robotic surgeons. Demographics of the participants are shown in Table 1.

\section{Performance evaluation and outcome measures}

The simulation was executed via the MentorLearn courseware which provides metrics and benchmark feedback for the simulation upon completion of a task. Outcome measures were based upon 25 provided and validated performance metrics including 11 general metrics such as task length and instrument collision as well as 14 task-specific metrics such as injury to the urethra and accuracy of the suturing technique (Table 2). The simulator we used did not have the possibility for participants to break the suture, and so this 25th metric is not included in the study.

\section{Comparison of participants}

We compared the performance of participants with low video game experience to performance of those with higher video game experience. Participants were divided into two groups: gamers with prior $\geq 6 \mathrm{hrs} /$ week of video game play and nonamers with prior $<6$ hrs/week. This way of measuring prior video game play experience has been used by several previous studies. $4,9,10$

Our definition of prior video game experience does not include the present amount of video game play but only the 
Table I Demographics of participants

\begin{tabular}{|c|c|c|c|c|}
\hline & Total NO & Gamers NO & Nongamers NO & $p$-value \\
\hline Number (NO) of participants & 32 & 12 & 20 & \\
\hline Median age (IQR) years & $25(24-26)$ & $24(23.8-25)$ & $25(24-26)$ & 0.111 \\
\hline Male/female & $15 / 17$ & $10 / 2$ & $5 / 15$ & \\
\hline Handedness (left/right/ambidextrous) & $4 / 26 / 2$ & $2 / 8 / 2$ & $2 / 18 / 0$ & \\
\hline Medical student/intern & $30 / 2$ & $11 / 1$ & $19 / 1$ & \\
\hline Median year of study (IQR) & $3.5(3.5-4.5)$ & $3.5(3.5-3.8)$ & $3.5(3.5-4.5)$ & 0.732 \\
\hline Experience with simulated surgery* $(\mathrm{Y} / \mathrm{N})$ & $8 / 24$ & $4 / 8$ & $4 / 16$ & \\
\hline VR game experience $(\mathrm{Y} / \mathrm{N})$ & $4 / 28$ & $3 / 9$ & $1 / 19$ & \\
\hline Prior video gameplay in hours/week (IQR) & $3.9(0.6-13)$ & $17(|2.8-2| .4)$ & I (0-3) & 0.004 \\
\hline
\end{tabular}

Notes: *Porcine models, VR (not robotic surgery), box-trainer, physical models.

Abbreviations: $Y$, yes; $N$, no; IQR: interquartile range; VR, virtual reality.

Table 2 Comparison of performance metrics between gamers and non-gamers

\begin{tabular}{|c|c|c|c|c|c|}
\hline \multirow[t]{2}{*}{ Performance metrics (unit) } & \multicolumn{2}{|l|}{ Gamers } & \multicolumn{2}{|c|}{ Nongamers } & \multirow[t]{2}{*}{$p$-value } \\
\hline & Median & IQR & Median & IQR & \\
\hline \multicolumn{6}{|l|}{ Time and economy } \\
\hline Time, s & 3728.5 & $3055-4698.3$ & 4229.5 & $3815-4869.5$ & 0.432 \\
\hline Movements of left ins., NO & 2122.5 & $2038.3-2443$ & 2571.5 & $2288.8-3450.8$ & 0.012 \\
\hline Movements of right ins., NO & 2504 & $2165.8-3074.8$ & 2996.5 & $2633.8-3720.3$ & 0.103 \\
\hline Path length of left ins., mm & $20,178.0$ & $17,558.5-22,132.6$ & 25,304 & $20,375.4-36,77 I .8$ & 0.015 \\
\hline Path length of right ins., $\mathrm{mm}$ & $21,920.9$ & $|8,89| . \mid-25,208.5$ & $28,473.2$ & $22,724.5-34,211.2$ & 0.063 \\
\hline Distance by camera, $\mathrm{mm}$ & 3905.1 & $2598-5473$ & 4628.8 & I522.3-5829.5 & 0.527 \\
\hline Instrument collisions, NO & 152.5 & $94.8-213$ & 199 & |46.8-273.2 & 0.081 \\
\hline Path instruments are out of view, $\mathrm{mm}$ & 4880.9 & I 727.5-7437.2 & 4266.7 & $2957.3-9213.7$ & 0.161 \\
\hline Times instruments are out of view, $\mathrm{NO}$ & 208 & $108-355.8$ & 210 & $93-328.5$ & 0.527 \\
\hline Time instruments are out of view, s & 564.5 & $143.5-1234$ & 463 & $279-1111.3$ & 0.578 \\
\hline Clutch usage, NO & 25 & $20.8-32.8$ & 26.5 & $18-53.5$ & 0.618 \\
\hline \multicolumn{6}{|l|}{ Safety and complications } \\
\hline Misapplied energy, NO & 0 & 0 & 0 & 0 & 0.790 \\
\hline Injury to urethra NO & 16 & $|1.5-3| .5$ & 17.5 & $10.8-35.8$ & 0.192 \\
\hline Injury to bladder neck, NO & 3 & $2-4.8$ & 4 & $2-8.5$ & 0.211 \\
\hline Suspected injury to bladder, NO & 0 & 0 & 0 & $0-1$ & 0.104 \\
\hline Injury to the NVB, NO & 0 & $0-3.8$ & 3,5 & $1.8-8$ & 0.071 \\
\hline Respect for tissue NO & 13 & $7.3-27$ & 14 & $8.5-35.3$ & 0.046 \\
\hline Suspected injury to the UO, NO & I & $0.8-2$ & 1 & $\mathrm{I}-2$ & 0.599 \\
\hline Suspected injury to the EF/US, NO & 10.5 & $4.5-16.3$ & 13 & $6.8-18.5$ & 0.442 \\
\hline Wound separation, mm & 2.09 & $1.05-3.14$ & 2.09 & $1.05-3.14$ & 0.652 \\
\hline Improper suturing technique, NO & 2 & $0-4$ & 3 & $\mathrm{I}-5.5$ & 0.134 \\
\hline \multicolumn{6}{|l|}{ Needle handling } \\
\hline Percentage of stitches in optimal depth & 56.6 & $52.6-75$ & 62.3 & $55.9-69.8$ & 0.388 \\
\hline Unnecessary needle piercing points, NO & 134.5 & $80-194.3$ & 182 & $101.8-252.8$ & 0.234 \\
\hline Entrance and exit points for needle, NO & 52 & $49.5-58$ & 56 & $51.5-60.5$ & 0.136 \\
\hline
\end{tabular}

Note: $P$-values for metrics showing significant differences are marked with bold.

Abbreviations: ins., instrument(s); NVB, neurovascular bundle; UO, urethral orifice; EF, endopelvic fascia; US, urethral sphincter; NO, number; IQR: interquartile range.

amount played somewhere in the past of the participants' lives. This time in life may vary between participants, as the questionnaire did not specify age. Moreover, performance between genders was compared. 


\section{Equipment}

The RobotiX Mentor ${ }^{\mathrm{TM}}$ (3D Systems; Simbionix Products, Cleveland, $\mathrm{OH}$ ) system is a VR simulator using the da Vinci interface. The platform consists of an adjustable headset with integrated stereoscopic visors, nonfixed hand controls, and foot pedals all integrated into a single console. It is a novel and latest high-fidelity simulator for assessment and training of trainees in robotic surgical skills with validation for this use. ${ }^{2,11,12}$

\section{Process}

We evaluated the influence of prior video game experience on VR simulation performance using the module "Guided urethrovesical anastomosis" (Figure 1) on the high-fidelity RM. Anastomosis is the last part of a robot-assisted radical prostatectomy, and the simulation displays the anatomy within pelvis after the prostate has been dissected and removed.

We performed all testing during daylight hours with slight background noise from personnel at both testing sites. Participants were guided orally for 5 mins about the hand controls, foot pedals and movement of the camera, according to a written standardized instruction. They were familiarized with the simulator using two modules for robotic basic skills. The first task "Camera 0" and the second task "Wristed manipulation level 2" were both tried once each, and participants were guided orally if in doubt. No data were collected during these modules. Participants then completed the module "Guided urethrovesical anastomosis" following a step-by-step instruction given by the simulator while it simultaneously collected

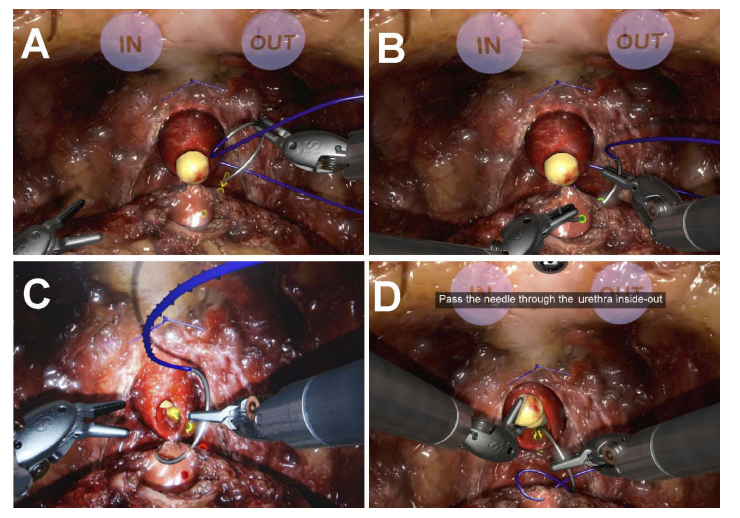

Figure I Guided urethrovesical anastomosis on RobotiX Mentor. The initial target point for guided sewing is marked with yellow and with an arrow indicating the direction (A). A green target point designates accurate needle puncture (B), whereas a red target point designates inaccurate needle puncture (C). The guided task provides step-by-step instructions (D). data. On the screen, yellow target points would appear to show where the suture should be put and a yellow arrow to indicate the sewing direction (Figure 1). The task was complete when the participant had put 5 stitches on either side of the anastomosis (ie, left and right). This module alongside with the simulator is described more thoroughly in a previous study. ${ }^{13}$ If in doubt, participants were guided orally by an observer blinded to their amount of prior video game experience.

Upon completion of the module, participants fulfilled a questionnaire gathering demographics, prior experience with simulated surgery, handedness, experience with VR games, console games and PC-games (Table 1).

\section{Statistical analysis}

We stored all questionnaire data and data from MentorLearn courseware anonymously in a specially designed Excel 2011 spreadsheet (Microsoft Corp., Redmond, WA, USA).

Data were analyzed using SPSS for MAC, version 24 (IBM Corp., Armonk, NY, USA) and Microsoft Excel 2011 (Microsoft Corp.). We considered a $p$-value $\leq 0.05$ significant.

\section{Results}

\section{Participants}

Thirty-three medical students and two intern volunteers (19 females; 16 males) participated in this study. One was excluded due to prior experience with VR robotic surgery and two were excluded due to technical difficulties during the simulation.

Finally, 30 medical students and two intern volunteers (17 females; 15 males) with a median age of 25 years (range, 24-26 years) participated in this study (Table 1). They were collected through convenience sampling in the period January to April 2017. All but one had passed their third year of medical school.

The group of nongamers $(\mathrm{N}=20)$ was almost twice as big as for gamers $(\mathrm{N}=12)$. Males were overrepresented among gamers and females were overrepresented among nongamers.

\section{Performance outcome for participants}

The gamers significantly outperformed the nongamers in 3 of 24 performance metrics $(p<0.05)$, which included "Number of movements of left instrument", "Path length of left instrument" and "Respect for tissue" (Table 2, Figures 2 and 3). For the remaining 21 metrics, there was no difference between the groups. It was not possible 


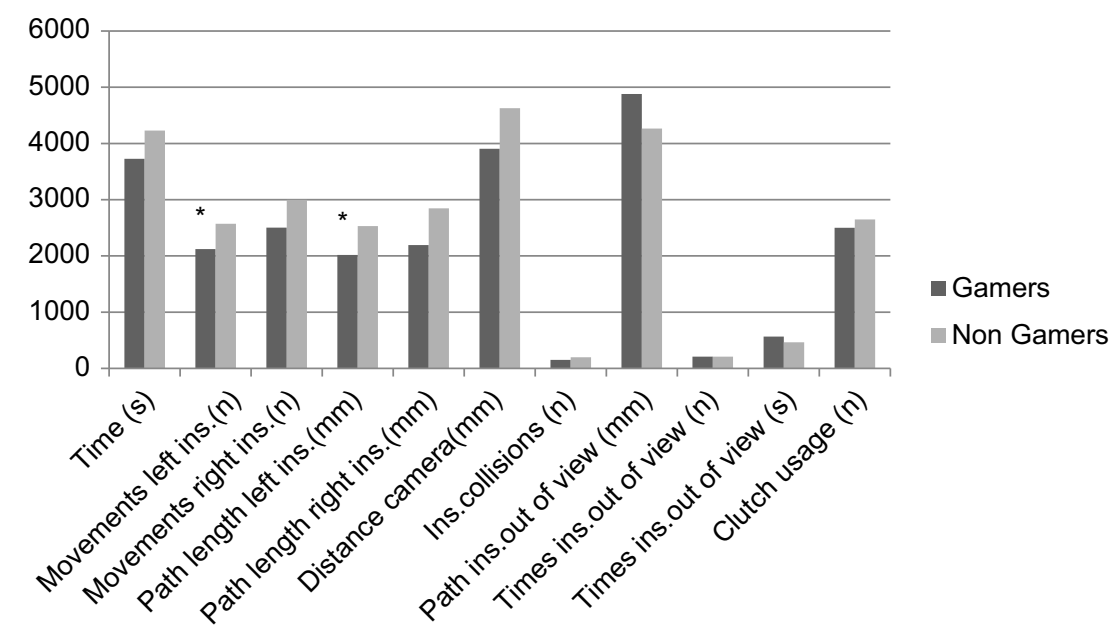

Figure 2 Mean performance metrics for Time and economy. Time is measured in second; path length left/right ins. measured in $10^{2} \mathrm{~mm}$. Distance camera and path ins. out of view measured in $\mathrm{mm}$. Clutch usage measured in numbers*10-2. Metrics showing significant difference are marked with $*$.

Abbreviation: ins., instrument(s).

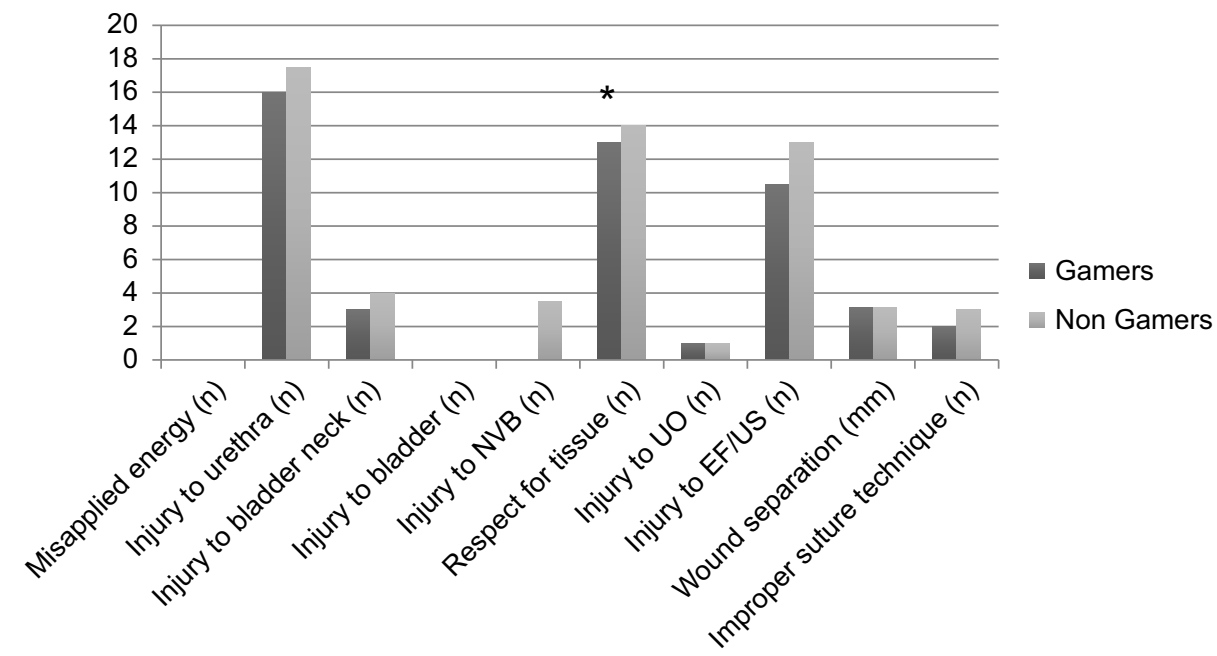

Figure 3 Mean performance metrics for safety and complications. Metrics showing significant difference are marked with *. Abbreviations: NVB, neurovascular bundle; UO, urethral orifice; EF, endopelvic fascia; US, urethral sphincter.

in the simulation to break the suture, and therefore both groups have a total of 0 occurrences. "Suture breakage" is for the same reason left out.

When comparing gender, males significantly outperformed females in 5 of 24 performance metrics ("Time", "Number of movements of left instrument", "Path length of left instrument", "Injury to the bladder" and "Injury to the neurovascular bundle"), but not after adjustment for experience with video games.
When comparing males in the two groups, male gamers significantly outperformed the male nongamers in 1 of 24 performance metrics ("Injury to the neurovascular bundle").

We could not compare females in the two groups since there are only 2 female gamers. The same accounts for handedness since there are only 4 left-handed and 2 ambidextrous.

Seven of the 24 performance metrics were normally distributed, and multiple regressions were conducted to 
compare the adjusted effect of being a gamer with being male. Males showed only significantly better performance in "Percentage of stitches within optimal depth" when adjusted for gaming experience.

\section{Discussion}

We demonstrated a difference in performance among medical students with $\geq 6 \mathrm{hrs} /$ week of game play compared to medical students with $<6 \mathrm{hrs} /$ week of game play. Gamers performed significantly better in 3 of 24 metrics ("Number of movements of left instrument", "Path length of left instrument" and "Respect for tissue") and showed better results for the majority of the remaining metrics (Figures 2-3). At best, nongamers performed just as good as gamers in the "Safety and complication metrics" (Figure 3). This group of metrics especially requires motoric skills and control of the robotic arms as well as visuospatial skills. However, our study is not robust enough to conclude that this difference in single parameters has any relevance, but the role of video gaming is very relevant when making educational curriculum for future surgeons. A recent paper proposes a new standard in analyzing and reporting VR outcome data - the Heidelberg VR score. ${ }^{14}$ The scoring algorithm shows the results on the importance of different skill aspects in laparoscopic surgery are universially applicable and that it can be transferred to any simulator or task. By incorporating specific expert baseline data for the respective task, one will be able to compare between tasks, studies and simulators.

The present study performing a vesicourethral anastomosis (VUA) as in robotic prostatectomy is a challenging task for novices due to delicate tissue and difficult suturing angles. A recent study was testing 10 surgical residents without prior robotics training where 5 residents received structured VR training, 5 did not and 4 were faculty robotic surgeons "experts". They found mean (range) completion percentage was $20 \%(10-30 \%), 54 \%(40-70 \%)$ and $96 \%(85-100 \%)$ by the untrained, trained and expert groups, respectively. ${ }^{15}$ Anastomosis integrity was rated as excellent (as opposed to moderate or poor) in $40 \%, 60 \%$ and $100 \%$ of untrained, trained and expert groups, respectively. It would have been interesting to know whether some of these had previous gamer experience which then could support our study. On-gamers sewed better in terms of "Percentages needle is in optimal depth". However, given their higher number of "Unnecessary needle piercing points", gamers might have been easier satisfied with the position of the needle even though the needle was not in optimal depth (red target point, Figure 1C), whereas nongamers have tried to perform the needle puncture as good as possible, piercing more times to get the needle in optimal depth (green target point, Figure 1B). Gamers had their instruments out of view for a longer time and a longer path, which is considered a lesser performance (Figure 2). But given the better performance in the "Safety and complication metrics", they might have been more aware of the instrument position even though not having them in view (Figure 3).

Previous studies have a tendency of asking whether video game experience improves surgical skills even though participants are only tested on simulators which do not resemble real-time surgery and which are only validated for the purpose of training basic motoric skills. Considering the studies showing a correlation between video game play and simulator performance, this might mean one of two things. First, it has been shown that practicing on a VR simulator improves real-time theater skills and therefore video game playing might do as well. Second, VR simulators are similar to video games, using the same principles; therefore, video game players have an advantage due to familiarity with the VR concept. ${ }^{16}$

Testing laparoscopic skills on LapSim ${ }^{\circledR}$ VR simulator, van Dongen et al found a significant difference in terms of the total score, efficiency and speed scores between interns with different levels of prior video game experience but not between school children with different video game experience. ${ }^{17}$ Neither the group of interns nor the school children differed in scores concerning precision. Unlike our findings, performance did not differ between genders.

Using VR laparoscopy on a MIST-VR, Grantcharov et al showed a correlation between surgeons' prior video game experience and less errors but not the time taken or numbers of unnecessary movements. ${ }^{18}$ They did not find any difference in performance between gender.

Willis et al tested medical students using VR and a physical model. ${ }^{19}$ They found a correlation between performance on Nintendo Wii and VR but not between Nintendo Wii and the physical model. Nor did the performance on VR correlate with performance on the physical model.

Earlier studies have tested the effect of video game experience on simulated robotic skills as the primary outcome, using the da Vinci robot. ${ }^{6,20}$ They did not find prior video game experience to be of any aid in simulated robotic surgery, but experience with different sports and skills of playing musical instruments seemed to benefit the robotic naive. Because the da Vinci robot uses an intuitive 
interface, the authors suggested that activities developing manual dexterity and intuitive hand-eye coordination such as sports and music could facilitate a more rapid acquisition of the skills and coordination necessary for robotic surgery. The intuitive interface in robotic surgery is different from laparoscopic surgery. Counterintuitive 3D motions are transferred to a $2 \mathrm{D}$ video screen, requiring the surgeon to sometimes work against the camera and perform complex procedures with the instrument moving on the projected video image opposite to what may seem natural. This type of movement may mimic what takes place during certain video games and hence aiding the laparoscopic naive but not the robotic naive. Their outcome measures were based upon skills of knot tying, and as known, there are many other skills required for the performance of robotic surgery. A randomized study was performed in order to evaluate the benefits of a combined multimodality training program for surgical residents with laparoscopic cholecystectomy. The Global Operative Assessment of Laparoscopic Skills (GOALS) showed training had improved GOALS scores while control did not. ${ }^{7}$ Another study explored the potential advantages of sequential learning of psychomotor and visuospatial skills for laparoscopic suturing and knot tying compared to simultaneous learning. ${ }^{8}$ They found after the laparoscopy-naïve medical students were randomized into a sequential learning group (SEQ) or a simultaneous learning group (SIM) that SEQ was faster than SIM at the beginning of the learning curve, but SEQ did not reduce the total training time needed to reach a proficiency level. However, SEQ needed less training on the box-trainer.

There are 6 VR simulators available for robot-assisted surgery; the da Vinci skills simulator, the mimic dV trainer, the ProMIS simulator, the simsurgery educational platform simulator, the robotic surgical simulator and the RM. Their efficacy is limited by the lack of comparative studies, validation and high cost. There is a need for a standard in analyzing and reporting VR outcome data, eg, the Heidelberg score. ${ }^{14}$ These simulators have to be incorporated into the urology training curriculum globally to shorten the learning phase. ${ }^{11,12}$

There are several limitations in our study. First, we had a study population, with a skewed gender distribution in our two groups, which made it difficult to assess whether the better performance was due to gender or due to prior video game experience. We used a new high-fidelity robotic simulator, the RM, and had no possibilities to use other simulators. Furthermore, participants varied in the type of games being played and the type of gaming unit(s) used.

Finally, a common denominator in the articles included from our search is the assessment of laparoscopic and robotic skills, which takes place on a simulator. Hence, even if a relationship between performance and video game playing is established, it will only refer to simulated performance. We suggest a future randomized controlled trial assessing the impact of video game playing on robotic skills in a theater.

Our study has several important implications and shows the need for unifying robotic surgery training curriculum that combines both basic skills development and procedure-specific training. The European Association of Urology and America Urological Association have identified and implemented a comprehensive training curricula with a 12-week training curriculum for robotic surgery, including VR, dry laboratory and wet laboratory simulations of all steps of the robotic prostatectomy. ${ }^{21,22}$

We used a high-fidelity simulator which was not the case in most previous studies. This simulator has shown to be useful and might offer the most authentic scenario for a VUA in robotic naive doctors to be tested on except for the expensive and ethical difficult porcine models and human cadavers. Furthermore, the RobotiX measures a broad variety of parameters and have shown its usefulness in the recent study by Shee. ${ }^{15}$

To our knowledge, this study is the first to evaluate the effect of prior video game experience on complex tasks in high-fidelity VR simulated robotic surgery. Future studies should agree on a common definition of prior video game experience, and a common way to measure performance outcome.

\section{Conclusion}

We demonstrated that prior video game experience might give an advantage in performing high-fidelity VR-simulated robotic surgery. We recommend future randomized studies with larger populations and common validated methods in order to incorporate video games in simulator programs for training and certification of robotic surgeons.

\section{Acknowledgment}

The authors would like to thank 3D Systems for the use of images of the simulated guided task.

\section{Disclosure}

The authors report no conflicts of interest in this work. 


\section{References}

1. Schreuder HWR, Persson JEU, Wolswijk RGH, Ihse I, Schijven MP, Verheijen RH. Validation of a novel virtual reality simulator for robotic surgery. ScientificWorldJournal. 2014;2014:507076.

2. Whittaker G, Aydin A, Raison N, et al. Validation of the RobotiX mentor robotic surgery simulator. $J$ Endourol. 2015;30:338-346.

3. Aydin A, Shafi AMA, Khan MS, Dasgupta P, Ahmed K. Current status of simulation and training models in urological surgery: a systematic review. J Urol. 2016;196:312-320.

4. Jalink MB, Goris J, Heineman E, Pierie JP, Hoedemaker C. The effects of video games on laparoscopic simulator skills. Am J Surg. 2014;208:151-156. doi:10.1016/j.amjsurg.2014.01.014

5. Aydin A, Raison N, Khan MS, Dasgupta P, Ahmed K. Simulationbased training and assessment in urological surgery. Nat Rev Urol. 2016;13:503-519.

6. Harper JD, Kaiser S, Ebrahimi K, et al. Prior video game exposure does not enhance robotic surgical performance. $J$ Endourol. 2007;21:1207-1210.

7. Kowalewski KF, Garrow CR, Proctor T, et al. LapTrain: multi-modality training curriculum for laparoscopic cholecystectomy-results of a randomized controlled trial. Surg Endosc. 2018;32(9):3830-3838. doi:10.1007/s00464-018-6110-7

8. Nickel F, Hendrie JD, Kowalewski KF, et al. Sequential learning of psychomotor and visuospatial skills for laparoscopic suturing and knot tying-a randomized controlled trial "the shoebox study" DRKS00008668. Langenbecks Arch Surg. 2016;401(6):893-901. doi:10.1007/s00423-016-1421-4

9. Rosser JC, Lynch PJ, Cuddihy L, Gentile DA, Klonsky J, Merrell R. The impact of video games on training surgeons in the 21 st century. Arch Surg. 2017;142:181-186.

10. Kennedy AM, Boyle EM, Traynor O, Walsh T, Hill ADK. Video gaming enhances psychomotor skills but not visuospatial and perceptual abilities. JSURG. 2011;68:414-420.

11. Watkinson W, Raison N, Abe T, et al. Establishing objective benchmarks in robotic virtual reality simulation at the level of a competent surgeon using the RobotiX mentor simulator. Postgrad Med J. 2018;94(1111):270-277. doi:10.1136/postgradmedj-2017-135351
12. MacCraith E, Forde JC, Davis NF. Robotic simulation training for urological trainees: a comprehensive review on cost, merits and challenges. J Robot Surg. 2019;13(3):1-7.

13. Setty Y, Salzman O. A methodological, task-based approach to procedure-specific simulations training. Int J Comput Assist Radiol Surg. 2016;11:2317-2324.

14. Schmidt MW, Kowalewski KF, Schmidt ML, et al. The Heidelberg VR score: development and validation of a composite score for laparoscopic virtual reality training. Surg Endosc. 2018. Epub 16 Oct 2018.

15. Shee K, Koo K, Wu X, Ghali FM, Halter RJ, Hyams ES. A novel ex vivo trainer for robotic vesicourethral anastomosis. J Robot Surg. 2019. Epub 28 Jan 2019.

16. Glassman D, Ishii H, Somani BK, Ahmed K, Biyani CS. Effect of playing video games on laparoscopic skills performance. J Endourol. 2016;30:146-152.

17. van Dongen KW, Verleisdonk EMM, Schijven MP, Broeders IAMJ. Will the Playstation generation become better endoscopic surgeons? Surg Endosc. 2011;25:2275-2280.

18. Grantcharov TP, Bardram L, Funch-Jensen P, Rosenberg J. Impact of hand dominance, gender, and experience with computer games on performance in virtual reality laparoscopy. Surg Endosc Other Interv Tech. 2003;17:1082-1085.

19. Willis RE, Gomez PP, Ivatury SJ, Mitra HS, Van Sickle KR. Virtual reality simulators : valuable surgical skills trainers or video games? $J$ Surg Educ. 2014;71:426-433.

20. Moglia A, Ferrari V, Morelli L, et al. Distribution of innate ability for surgery amongst medical students assessed by an advanced virtual reality surgical simulator. Surg Endosc Other Interv Tech. 2014;28:1830-1837.

21. Ahmed K, Khan R, Mottrie A, et al. Development of a standardised training curriculum for robotic surgery: a consensus statement from an international multidisciplinary group of experts. BJU Int. 2015;116(1):93-101.

22. Smith R, Patel V, Satava R. Fundamentals of robotic surgery: a course of basic robotic surgery skills based upon a 14-society consensus template of outcomes measures and curriculum development. Int J Med Robot. 2014;10(3):379-384.
Advances in Medical Education and Practice

\section{Publish your work in this journal}

Advances in Medical Education and Practice is an international, peerreviewed, open access journal that aims to present and publish research on Medical Education covering medical, dental, nursing and allied health care professional education. The journal covers undergraduate education, postgraduate training and continuing medical education including emerging trends and innovative models linking education, research, and health care services. The manuscript management system is completely online and includes a very quick and fair peer-review system. Visit http://www.dovepress.com/testimonials.php to read real quotes from published authors. 